\title{
Saguenay 2025 : \\ les enjeux des changements climatiques
}

\author{
Claude Villeneuve ${ }^{1}$ \\ Université du Québec à Chicoutimi
}

\section{Introduction}

Le présent article vise à illustrer comment les changements climatiques risquent de transformer à moyen terme de nombreuses caractéristiques de la région du Saguenay-Lac-Saint-Jean et affecter possiblement ses perspectives de développement si la région maintient la prédominance des ressources naturelles comme moteur de son activité économique. En effet, tant dans le domaine de l'agriculture que de la forêt, dans la production d'hydroélectricité, la production d'aluminium et de papier, dans le tourisme ainsi que dans la gestion du risque et l'établissement des primes d'assurance, les changements climatiques vont rendre plus aléatoire la prévision des futurs possibles.

\section{La variabilité climatique}

Le climat représente une moyenne de températures et de précipitations qui caractérise une région. Pour les êtres vivants, cela constitue une donnée fondamentale car, ne l'oublions pas, tant les plantes que les animaux vivent dehors à l'année longue et doivent s'adapter aux variations locales de ces facteurs fondamentaux, sous peine de disparaître. Ainsi, lorsqu'on choisit des plantes pour son jardin, on s'intéresse à ce qu'elles soient suffisamment rustiques pour la zone où l'on souhaite les voir pousser. De la même façon, la répartition des précipitations d'une saison à l'autre, et au cours d'une même saison, détermine la croissance des forêts ainsi que la présence et l'abondance des espèces animales. Même si les humains se sont affranchis des obligations biologiques de l'adaptation aux variations climatiques, un grand nombre de nos activités de production sont influencées directement par le climat et ses extrêmes.

Les inondations de 1996 au Saguenay nous ont bien montré ce que voulaient dire des précipitations extrêmes, mais savons-nous ce que représente pour nous le climat? Dans la région, la production d'hydroélectricité, donc d'aluminium, la croissance des forêts, le rendement de l'agriculture et même le tourisme dépendent de la prévisibilité du climat et de son maintien autour des normales. Les années exceptionnellement sèches ou les crues très abondantes occasionnent des pertes importantes non seulement en fonction des pertes directes, mais aussi en raison de la difficulté au cours des années suivantes à gérer cette variabilité. En effet, notre gestion se base sur un historique, et les années où le climat connaît des extrêmes imposent par la suite des marges de sécurité et des décisions qui doivent tenir compte de ce type d'événements. La construction du barrage Pikauba et les nouvelles normes de gestion du lac Kénogami montrent que cela peut avoir des coûts monétaires, environnementaux et sociaux.

Le climat représente des moyennes autour desquelles se trouvent des données probables réparties sur la courbe normale de Laplace-Gauss. On s'approche ou l'on s'éloigne de la moyenne selon une probabilité qui est à l'inverse de l'amplitude de l'écart. Ainsi, si la température moyenne de juillet a été de 20 degrés sur la moyenne des trente dernières années, on considérera qu'un mois de juillet dont la moyenne est de 20,3 degrés est presque normal et peut se produire une année sur trois, tout comme les années à 19,7 degrés. Par contre, des mois de juillet avec une moyenne de 25 ou de 15 degrés sont beaucoup plus rares et impro- 
bables. Ils se produisent, disons, une année sur cent. Ces moyennes cachent pourtant la réalité, car elles incluent les températures du jour et de la nuit. Un mois où l'air est sec et le ciel ensoleillé va donner une moyenne de 20 si les jours sont à 30 degrés et les nuits à 10 ; ce sera la même moyenne pour un mois nuageux et pluvieux où les températures sont de 22 le jour et de 18 la nuit. Cette variabilité du climat, nous l'observons à chaque jour et les moyennes ou les normales nous la cachent.

Si notre position par rapport à l'équateur détermine la variation de la durée du jour, donc de la quantité d'énergie solaire reçue en un point du globe, la répartition des précipitations est influencée par un grand nombre de facteurs locaux et régionaux tels la proximité des masses d'eau, le relief et autres facteurs comme la présence de forêts. La combinaison de l'ensoleillement, de l'abondance et de la répartition des précipitations détermine le climat d'une région et le régime des crues de son bassin hydrographique.

\section{Dans d'autres endroits, les changements climatiques se traduisent par une augmentation des temps violents, par des sécheresses inhabituelles ou par des canicules comme celle qu'on a observée en Europe à l'été 2003.}

\section{Les changements climatiques}

Depuis la troisième série de rapports du Groupe intergouvenemental d'experts sur le climat (GIEC) en 2001, la communauté scientifique dans son ensemble fait consensus sur l'origine anthropique d'un vaste changement de la composition de l'atmosphère à l'échelle du globe par l'accumulation de gaz à effet de serre. Cette pollution de l'atmosphère se traduit par une augmentation de la température planétaire qui risque de progresser de plus de deux degrés au $21^{\mathrm{e}}$ siècle dans un scénario de doublement de la quantité pré-industrielle de ces gaz. Cette situation devrait se produire avant 2050 selon les scénarios actuels. Déjà, au vingtième siècle, on a enregistré une hausse de 0,6 degrés Celsius de la moyenne planétaire. Cette hausse est beaucoup plus forte et rapide que tout ce qu' on a pu observer dans le dernier millénaire ${ }^{2}$. Les experts prévoient que, même si tout était fait immédiatement pour arrêter l'augmentation des gaz à effet de serre dans l'atmosphère, on assisterait quand même à une augmentation de 0,75 degrés supplémentaires d'ici 2050 en raison de l'inertie du système et des quantités de GES déjà émis. Cette augmentation est donc inévitable et se produira vraisemblablement plus rapidement que prévu puisque les mesures visant à réduire cette forme de pollution ne sont pas encore en place, loin s'en faut.

Ces modifications, en apparence petites à l'échelle globale, peuvent s'avérer fort importantes à l'échelle locale. Par exemple, dans le nord du Canada, on a observé dans la région du McKenzie une augmentation de la température moyenne de près de 6 degrés Celsius depuis le début des années 1980. Cette augmentation se traduit par des changements importants dans la vie des populations locales tels que la fonte du pergélisol, la difficulté de faire des chemins de glace en hiver, etc. L'augmentation de la température moyenne se traduit surtout par une augmentation des températures moyennes en hiver (moins de froids intenses, redoux inopinés) et la nuit en été.

Dans d'autres endroits, les changements climatiques se traduisent par une augmentation des temps violents, par des sécheresses inhabituelles ou par des canicules comme celle qu'on a observée en Europe à l'été 2003. $\mathrm{Au}$ Québec, on associe aux changements climatiques les températures inhabituelles observées au cours des dix dernières années, la fonte du pergélisol dans le Nord et la baisse de niveau du fleuve Saint-Laurent. Dans les zones du littoral maritime, on observe une accélération de l'érosion attribuable à l'augmentation du niveau des océans prédit par le modèle. Il demeure beaucoup d'incertitudes sur l'ampleur des changements à l'échelle locale, la résolution des modèles utilisés étant encore insuffisante. Cependant, l'équipe de recherche en climatologie du consortium OURANOS a mis au point des modèles climatiques régionaux qui devraient nous donner des réponses à ce sujet dans les trois prochaines années ${ }^{3}$.

\section{La réaction politique}

Alertés par les scientifiques à la fin des années 1980 , les autorités politiques ont rapidement adopté la Convention cadre des Nations Unies sur les changements climatiques (CCNUCC) au terme du Sommet de Rio de 1992. Par la suite, les États ont ratifié la Convention en 1994 et ont commencé à en négocier l'appli- 
cation. L'objectif de la Convention était de s'attaquer au problème et de stabiliser en 2000 les émissions des pays développés au niveau de 1990. Naturellement, cela n'a pas été fait, malgré que l'effondrement de l'économie soviétique ait donné une marge de manœuvre de plus d'un milliard de tonnes d'émissions. Les Parties à la convention se sont donc entendues sur les principes généraux du Protocole de Kyoto en 1997 qui retardait l'horizon de la stabilisation à la période 2008-2012 et préconisait, pendant cette période, une réduction moyenne de $5 \%$ par rapport à l'année de référence 1990. Cela devait être atteint par des objectifs volontaires mais contraignants de réduction de la part des pays industrialisés, sans mesures particulières des pays en voie de développement.

Le Protocole de Kyoto est actuellement en panne compte tenu du refus des États-Unis et de l'Australie de le ratifier en 2001. Malgré que 140 pays l'aient sanctionné à ce jour, le défaut de la Russie de s'engager bloque la mise en œuvre de la convention puisque le Protocole doit être ratifié par des pays représentant $55 \%$ au moins des émissions des pays industrialisés pour entrer en vigueur. Cette situation risque de faire déraper sérieusement la négociation des engagements pour la deuxième période (2013-2017) de référence ${ }^{4}$.

Par ailleurs, pour beaucoup de pays - dont évidemment le Canada - le blocage de Kyoto a un effet ralentisseur sur les mesures concrètes à mettre en œuvre pour atteindre leurs objectifs. Pourquoi donc s'imposer des obligations dans une économie mondialisée alors que les concurrents américains, australiens et russes ne le font pas ? Sans compter que les pays en voie de développement ne sont pas contraints à des limitations de leurs émissions.

Il est donc vraisemblable que les seules chances que la communauté internationale s'engage rapidement dans la réduction des gaz à effet de serre tiennent à une élection présidentielle américaine qui amènerait un changement de cap à la Maison Blanche en 2004. La réélection de la présidence actuelle pour un autre mandat constituera un arrêt de mort pour le Protocole de Kyoto. Même dans le premier cas, il est peu probable que les résultats de Kyoto soient atteints puisque les principaux émetteurs ont pris du retard dans leurs engagements et que les pays en voie de développement, en particulier la Chine, l'Inde et le Brésil, ont continué d'augmenter leurs émissions.

Or, les scientifiques sont formels: les changements climatiques sont déjà commencés et la vitesse à laquelle leurs effets se produisent est plus rapide que ce que les modèles prévoient. Il faut donc considérer comme conservatrices les prévisions actuelles et se préparer à s'adapter à une modification majeure du climat au Québec et dans la région. L'inertie du climat planétaire est telle que le prochain siècle sera affecté de façon très importante, peu importe les actions entreprises dans les prochaines années. On espère au mieux stabiliser la concentration de dioxyde de carbone autour de 570 à 600 parties par million, ce qui voudrait dire, pour un pays comme la France, de couper ses émissions par quatre d'ici 2050 et, pour le Canada, par six ou huit de manière à laisser une marge de croissance pour les pays en voie de développement.

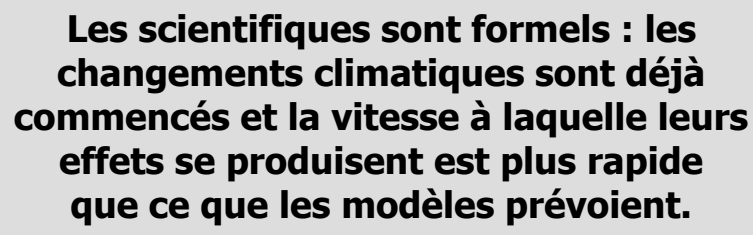

\section{Les prévisions des changements climatiques à l'échelle régionale}

Même s'il est peu probable que le Saguenay devienne une terre vinicole en 2025, Agriculture Canada ${ }^{5}$ prévoit néanmoins que le climat de Normandin au LacSaint-Jean sera aussi chaud que celui de SaintHyacinthe aujourd'hui, bien avant 2050. Plusieurs pourraient être tentés de se réjouir de la chose, mais rien n'est plus trompeur qu'une moyenne annuelle. Le réchauffement des moyennes cache une réalité très importante: le réchauffement sera plus perceptible par une hausse des minima, donc les températures nocturnes et les températures hivernales, que par les hausses des températures diurnes et estivales. Cela signifie aussi une augmentation de la variabilité climatique car, même si notre climat est globalement plus chaud, nous ne serons pas plus à l'abri des masses d'air froid venues de l'Arctique ou des systèmes chargés d'eau du golfe du Mexique. Enfin, les modèles climatologiques prévoient aussi une augmentation de la fréquence des temps violents et des blocages de systèmes comme celui qui nous a valu les inondations de 1996. Cette perspective est très inquiétante, car si les événements exceptionnels deviennent monnaie 
courante, il faudra surveiller le prix des assurances, tant pour les productions agricoles que pour protéger nos investissements immobiliers et industriels.

Dans la suite de ce texte, nous examinerons ce que cela veut dire dans divers secteurs de notre activité économique.

\section{La vulnérabilité des activités traditionnelles de la région de Saguenay}

Les régions du Saguenay et du Lac-Saint-Jean se caractérisent par une faible diversification des activités industrielles : production d'aluminium, de bois d'œuvre et de pâtes et papiers, agriculture et tourisme résument en gros les mamelles auxquelles s'abreuve la vitalité économique. L'essentiel de notre production est destinée à l'exportation et les marchés locaux sont intégrés aux réseaux de distribution qui dominent le continent nord-américain. En somme, la région est très dépendante des marchés extérieurs et possède peu de prise sur son propre développement. De plus, comme ailleurs au Québec, sa population est vieillissante et s'urbanise de plus en plus. Elle est donc davantage dépendante $\mathrm{du}$ bon fonctionnement des systèmes d'approvisionnement et des services.

Ce portrait peu reluisant pourrait se détériorer encore si les autorités compétentes ne prennent pas en compte les transformations, les risques et les occasions que laissent entrevoir les changements climatiques. Nous tenterons ici d'en dégager pour chaque secteur.

\section{Variabilité des précipitations et hydraulicité}

C'est le réseau hydrographique qui explique l'intérêt de l'industrie lourde pour la région. La production d'énergie hydroélectrique (le réseau d'Alcan à lui seul génère une puissance de 2000 mégawatts) se fait grâce aux grandes rivières du lac Saint-Jean qui drainent plus de 75000 kilomètres carrés et où il tombe en moyenne 120 centimètres de pluie annuellement. Ces précipitations expliquent aussi la présence de forêts qui ont supporté l'autre grande industrie régionale dans son développement.

Le régime des crues, avec ses fortes fluctuations saisonnières qui varient naturellement de l'ordre de 10 à 30 fois entre les débits d'étiage et les débits de crue, oblige à construire de grands ouvrages de retenue pour conserver l'eau des crues et en régulariser le débit en fonction de la demande hydroélectrique. Dans le cas d'une demande industrielle, celle-ci est constante toute l'année. Il est donc important de bien prévoir les pointes de crue pour gérer efficacement les barrages et ne pas perdre d'eau.

Dans un scénario de changements climatiques, les périodes de pluies intenses alterneront avec des sécheresses prolongées. En soi, cela peut être géré avec des outils efficaces de prévision et une capacité de stockage importante. Cependant, d'autres phénomènes viennent compliquer les problèmes potentiels. Des hivers plus chauds et des pluies hivernales abondantes, ou des redoux inopinés qui peuvent provoquer un manque d'eau printanier, sont des phénomènes qui risquent de compliquer sérieusement la gestion du réseau hydroélectrique et obliger peut-être la création de nouvelles capacités de stockage sur les grandes rivières du lac Saint-Jean. Connaissant les réticences de la population à de tels développements, la partie n'est pas gagnée d'avance. Par ailleurs, les installations d'Hydro-Québec en milieu nordique sont aussi menacées d'une pénurie d'eau. Cela oblige actuellement l'entreprise à envisager la construction de centrales au gaz naturel. Comment cela se traduira-t-il dans la fourniture et les prix de l'électricité pour les citoyens du Québec en 2025 ? Il s'agit encore d'une incertitude à laquelle nous ne pouvons apporter aucune réponse. Les travaux du consortium OURANOS sur ce sujet sont en phase de modéliser les précipita. tions dans trois bassins versants, dont celui du Saguenay comme pilote pour la modélisation d'autres bassins plus nordiques. Il est vraisemblable que ces travaux nous donneront des éléments de réponse sur ce sujet d'ici trois ou quatre ans.

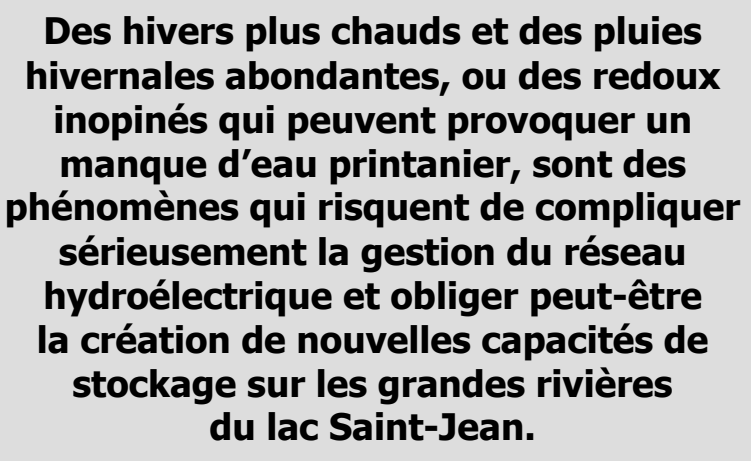




\section{Production de GES et droits d'émissions}

Un acquis qui restera vraisemblablement du Protocole de Kyoto est le mécanisme des permis échangeables. Ce mécanisme, qui permet aux entreprises et aux États de troquer des réductions d'émissions, permet la souplesse du marché dans les échanges d'avantages comparatifs entre les pays et les industries. Par exemple, une industrie qui utilise du charbon comme intrant dans un procédé et non pas comme source d'énergie ne peut pas réduire ses émissions aussi facilement qu'une entreprise qui fait, par exemple, le changement de sa source de carburant, passant du pétrole au gaz naturel. Que la première entreprise puisse acheter des crédits excédentaires de la seconde se traduit de toute façon par une réduction et permet à la seconde de rentabiliser plus rapidement ses investissements.

L'avantage de l'hydroélectricité régionale est à cet effet un modèle. Par exemple, une aluminerie alimentée avec de l'électricité produite à partir de charbon émettra la même quantité de gaz à effet de serre comme émission de procédés qu'une usine qui s'approvisionne à l'hydroélectricité, soit une moyenne de 3,7 tonnes par tonne d'aluminium produit. Cependant, la quantité de gaz à effet de serre totale émise est de 13,5 tonnes par tonne d'aluminium produite pour la production d'électricité par le charbon, alors qu'il est à peu près nul pour l'hydroélectricité. La plupart des alumineries aux États-Unis et en Chine fonctionnent avec de l'électricité produite à partir du charbon.

Une entreprise comme Alcan qui produit actuellement en moyenne 4 tonnes de gaz à effet de serre par tonne d'aluminium dans la région pourrait donc voir sa compétitivité augmenter par rapport à ses concurrentes américaines qui montrent un bilan beaucoup moins reluisant.

\section{Le principal facteur limitant la croissance des forêts est la disponibilité de l'eau au printemps.}

\section{Forêts et carbone}

La région est actuellement la plus grande région forestière du Québec. On y prélève bon an mal an environ 9 millions de mètres cubes de bois dont la majeure partie est transformée en bois d'œuvre. Or, le bois provient du gaz carbonique retiré de l'atmosphère par la photosynthèse des plantes. Le principal facteur limitant la croissance des forêts est la disponibilité de l'eau au printemps. Les printemps secs se caractérisent donc par des croissances forestières plus faibles dans la forêt boréale qui couvre la majeure partie de notre domaine forestier.

Par ailleurs, les travaux du Consortium de recherche sur la forêt boréale commerciale de l'UQAC ont démontré l'existence d'un mécanisme de déforestation naturelle qui affecte le domaine de la pessière noire ${ }^{6}$. $\mathrm{Ce}$ phénomène explique la présence de millions d'hectares de forêts ouvertes ou insuffisamment régénérées en raison de la récurrence des feux.

Même si certains auteurs présument que la croissance forestière pourrait être meilleure par la combinaison de l'augmentation de la température et de l'effet fertilisant de plus grandes quantités de $\mathrm{CO}_{2}$, cette hypothèse est loin d'être démontrée et surtout, nul ne peut prédire quel sera l'effet des changements climatiques sur la disponibilité de l'eau au printemps et l'incidence des incendies de forêt sur le territoire. Ces facteurs d'incertitude vont très certainement compliquer la planification des activités de récolte forestière et la prévision des rendements de la forêt au cours du prochain siècle.

En revanche, il est maintenant possible de considérer que la plantation de ces millions d'hectares de parterres forestiers non productifs ou mal régénérés pourrait devenir à court terme un immense projet de séquestration du carbone. En effet, la plantation de ces parterres pourrait constituer un potentiel de séquestration de l'ordre de 10 millions de tonnes de $\mathrm{CO}_{2}$ annuellement pour le Québec ${ }^{7}$, ce qui constituerait une compensation pour cinq fois les émissions de la centrale du Suroît. La production des milliards de plants et la main-d'œuvre nécessaire pour les mettre en terre et les protéger contre les feux représente une avenue inattendue d'emplois dans les villages forestiers du Saguenay et du Lac-Saint-Jean.

Finalement, comme le gouvernement du Canada a inscrit dans son plan d'action contre les changements climatiques l'objectif d'intégrer $10 \%$ d'éthanol dans $35 \%$ du carburant vendu au Canada d'ici 2010, la demande pour ce carburant produit à partir de la biomasse va augmenter de façon exponentielle. Il pourrait 
s'agir d'une activité économique intéressante pour des entrepreneurs capables de développer des usines mobiles de production de méthanol et d'éthanol à partir des résidus de coupe. En effet, à la fois au niveau économique et au plan des bilans de carbone, le transport des résidus vers les centres urbains s'avère improductif, alors que le transport du produit fini aurait un meilleur impact écologique.

\section{Agriculture : adieu gourganes !}

Dans le contexte actuel de mondialisation du commerce et de prépondérance des chaînes de distribution dans le commerce alimentaire, l'agriculture connaît une crise profonde, particulièrement face à la compétition des produits de masse. Par ailleurs, depuis toujours, les agriculteurs basent leurs décisions de production sur les résultats de la saison précédente. Dans ce contexte, l'augmentation de la variabilité climatique imposera à la Financière agricole des augmentations importantes de coûts qui l'inciteront sans doute à vouloir réduire ses risques. Dans ce contexte, les productions animales seront privilégiées en raison de leur plus faible susceptibilité au climat. Malheureusement, ces productions de plus en plus effectuées dans des conditions contrôlées exigent une gestion rigoureuse des fumiers pour être acceptable par les populations riveraines.

Le problème du drainage des terres est un exemple des complications que peut apporter le changement climatique. Au Québec, la saison estivale a toujours été la saison où les précipitations sont les plus abondantes (entre $40 \%$ et $60 \%$ ) des précipitations totales annuelles en trois mois. Si l'on ajoute à ce fait la fonte des neiges qui inonde les sols au printemps, les agriculteurs doivent le plus souvent drainer leurs terres pour en évacuer l'eau de manière à pouvoir produire plus efficacement. Dans un scénario de changement climatique où les précipitations sont plus violentes et sont suivies de périodes prolongées de sécheresse, les sols drainés seront trop secs pour certaines formes d'agriculture comme la production de foin et certaines cultures maraîchères. En outre, certains produits comme la gourgane demandent des climats frais et humides pour bien réussir. Il est impossible de cultiver cette fève dans la région de Montréal, par exemple. Si l'on se retrouve effectivement avec le climat de Saint-Hyacinthe en 2025, c'est une culture traditionnelle qu'il faudra oublier.

\section{Tourisme : lorsque l'hiver se fait rare}

Sur ce plan, deux facteurs sont à surveiller : le devenir des populations de saumons atlantiques et le tourisme hivernal. Par ailleurs, les événements de temps violent ou les précipitations exceptionnelles auront toujours, en raison de leur caractère médiatique, un effet rédhibitoire sur la fréquentation des attraits touristiques régionaux, comme on a pu s'en rendre compte à l'été 1996.

Le saumon atlantique est une espèce très fragile aux températures de l'eau dans les rivières, ce qui explique sa limite sud de répartition. En règle générale, les saumons doivent éviter des eaux dont la température dépasse $25^{\circ} \mathrm{C}$. Des étés plus chauds, des précipitations plus violentes favorisent les étiages sévères et les crues dévastatrices. Avec une telle modification du régime des eaux, on peut craindre que les rivières à saumons, qui justifient un intéressant tourisme estival, ne voient leur productivité réduite ou même une remontée vers le nord de l'aire de répartition de l'espèce.

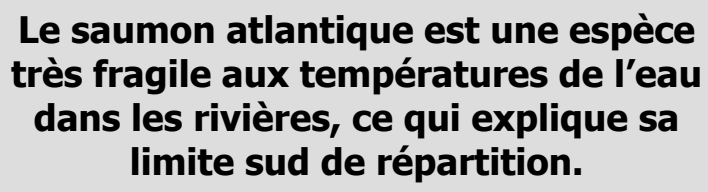

La modification la plus importante dans l'offre touristique viendra du tourisme hivernal. Le sud du Québec souffre déjà d'une grande incertitude dans les conditions nivales, et tant le ski de fond que le ski alpin ou la motoneige y sont difficiles à pratiquer pendant la majeure partie de l'hiver, une année sur deux. L'intensification du réchauffement devrait rendre ces activités très aléatoires, ce qui donnera de la valeur à l'offre régionale. En effet, malgré le réchauffement, nos centres de ski et nos sentiers de motoneige devraient continuer de disposer pour quelques décennies encore de conditions climatiques favorables.

\section{Quelles mesures prendre maintenant pour tirer avantage des changements climatiques?}

Comme nous l'avons vu rapidement dans les pages précédentes, il ne manque pas d'exemples de la vulné- 
rabilité de notre économie aux changements climatiques. Il existe même quelques possibilités sérieuses de tirer parti de cette situation, mais cela exige de savoir prévoir, d'anticiper le changement et de se donner les outils pour s'adapter. Parmi ces outils, certains sont à la portée des entreprises ou des gouvernements supérieurs, mais il importe de développer dans la population les aptitudes à l'innovation et à la créativité pour réduire notre dépendance envers les ressources naturelles et nous protéger de la variabilité croissante du climat. Dans le monde d'aujourd'hui, toute région qui fonde son développement uniquement sur ses ressources naturelles est vouée au sousdéveloppement durable.

\section{Aménager le territoire en prévision des crues}

Les dangers associés à la gestion des crues seront un enjeu majeur des prochaines décennies. La capacité de prévoir correctement les précipitations est une chose, mais l'aménagement du territoire pour éviter les dommages en est une autre. Cet aménagement préventif appartient totalement aux autorités régionales. Les principaux avantages d'un tel aménagement sont la protection des personnes et des investissements.

\section{Dans le monde d'aujourd'hui, toute région qui fonde son développement uniquement sur ses ressources naturelles est vouée au sous-développement durable.}

\section{Développer des entreprises axées sur l'efficacité énergétique}

L'énergie sera un enjeu majeur des prochaines décennies. Plusieurs auteurs estiment que le point d'inflexion dans la courbe des découvertes de réserves de pétrole versus la consommation sera bientôt atteint. À partir du moment où cela se produira (entre 2015 et 2025), les énergies renouvelables et l'efficacité énergétique prendront une valeur ajoutée compte tenu de l'inévitable augmentation du prix du pétrole à l'échelle mondiale. En conséquence, les entreprises qui auront développé une expertise et des pratiques dans ce domaine verront leurs produits et leur savoir-faire en meilleure position sur les marchés.

\section{Tirer avantage de nos forêts et combattre la déforestation naturelle}

Le bois utilisé dans la production de biens durables est un moyen efficace de séquestrer du $\mathrm{CO}_{2}$ qui est reconnu par la communauté internationale. C'est d'ailleurs un des éléments majeurs de la stratégie française de lutte aux changements climatiques. En améliorant l'efficacité de la transformation du bois en biens durables, en plantant des forêts dans les zones actuellement mal régénérées et en mettant sur pied des entreprises spécialisées dans la production de biocarburants à partir de la biomasse forestière résiduelle, on peut penser que la région pourra tirer des avantages supplémentaires de son patrimoine forestier.

\section{Développer des créneaux originaux de production}

Dans le monde de l'agriculture comme dans la production en général, les produits de deuxième et troisième transformation demandent un savoir-faire plus grand, mais génèrent des retombées plus importantes en termes d'emploi et de valeur ajoutée. Par ailleurs, des créneaux originaux de production sont à la portée de plus petites entreprises qui demandent moins de capitalisation. Dans le domaine des produits agricoles, la recherche de productions originales, voire uniques, permet d'occuper des créneaux où la valeur ajoutée des produits compense largement pour les frais liés à l'éloignement des marchés.

\section{Conclusion}

Les changements climatiques nous affecteront non seulement à l'échelle locale, mais aussi en fonction de la façon dont ils détermineront la demande pour nos produits et le coût de nos fournitures en provenance de l'extérieur. Nier cette réalité, c'est se condamner à subir les conséquences négatives sans espoir de tirer avantage des occasions d'occuper de nouveaux créneaux de développement pour la région. En ce qui a trait au climat, l'horizon 2025 demeure plein d'inconnues. Il est donc impératif d'investir dès aujourd'hui dans la recherche et la formation de manière à faire émerger les technologies et les projets qui nous permettront de relever ce défi au lieu d'attendre que d'autres s'en occupent à notre place. Vivre les changements climatiques n'est-il pas un projet plus motivant que d'y survivre? 


\section{Notes et références}

1 Directeur de la Chaire en Éco-Conseil, au département des sciences fondamentales de l'UQAC, l'auteur a reçu le prix de scientifique de l'année 2001 pour son livre Vivre les changements climatiques. Membre du cercle des Phénix de l'environnement, il a été fondateur de la Région laboratoire du développement durable et dirige depuis 2001 le programme de DESS en Éco-conseil.

2 Villeneuve, C. et F. Richard (2001). Vivre les changements climatiques. L'effet de serre expliqué, Sainte-Foy, Éd. MultiMondes, $274 \mathrm{p}$.

Beauchemin, Georges, Présentation aux éco-conseillers sur les travaux du Consortium OURANOS le 3 décembre 2003, Université du Québec à Chicoutimi.
4 Radanne, P. (2004), Introduction au séminaire de Montréal des 17 au 19 mars sur les négociations à venir sur les changements climatiques, IEPF, $30 \mathrm{p}$.

5 Bélanger, Gilles, chercheur, Agriculture et agroalimentaire Canada, communication faite le 4 février 2003 lors de la journée «Les changements climatiques et la rentabilité de votre entreprise agricole », Technopole Laval.

6 Consortium de recherche sur la forêt boréale commerciale (2000). Au royaume de la forêt boréale, Le groupe MADIE, $60 \mathrm{p}$.

7 Villeneuve, C. (2003). La séquestration du carbone, perspectives pour le Québec, mémoire présenté devant la commission parlementaire sur les transports et l'environnement publié en ligne à l'adresse : http://www.menv.gouv.qc.ca/ air/changement/kyoto/memoires/sequestration.pdf. 